\author{
I.D. GORBENKO, Dr. of Sc, O.A. ZAMULA, Dr. of Sc, V.L. MOROZOV, \\ S.V. RODIONOV, PhD

\section{MATHEMATICAL MODEL OF ORTHOGONAL FREQUENCY SIGNALS DISTRIBUTION AND MULTIPLEXATION (OFDM)}

\title{
Introduction
}

The need for new communication services is constantly increasing. At the same time the requirements for transmission speeds, noise-receiving of data, secrecy of functioning of the system, quality of services are provided. This leads to a worsening of the contradiction between the increasing requirements and the limited frequency resources, which in turn sets the task of increasing the spectral efficiency of the systems. It is known that a significant increase in pro-launch capability and reliability of communication can be achieved by using systems with multiple inputs and multiple outputs (MIMO), which uses multiple antennas on the transmitting side and multiple antennas on reception site. In combination with adaptive modulation and coding schemes, as well as the adaptive allocation of system resources, these methods can provide significant improvements in data rates and communication reliability [1]. Studies have shown that both MIMO technologies and other spectral efficiency enhancement techniques can be used in conjunction with multi-carrier transmission technologies, in particular, MIMO-OFDM (orthogonal frequency division multiplexing, hereinafter OFDM) and MIMO-OFDMA (orthogonal) technologies frequency division multiple access). Being able to provide subscribers with a wide range of applications with different capabilities in terms of tolerable delay, quality of service, bandwidth requires future systems of high resistance to interference and channel distortion, as well as more flexibility in radio resource management. Choosing the right radio interface is key to ensuring these properties of the communication system. Multiple Carrier Technology in orthogonal frequency division multiplexing is widely recognized as one of the most promising access schemes for use in advanced wireless communication systems

\section{The main results of the research}

The basic idea of OFDM is to split high-speed data flow into a number of sub-streams at lower speeds [1]. These sub-streams are then transmitted in parallel orthogonal subchannels, resulting in partial overlap of the spectrum. Compared to single-carrier transmission, this approach provides increased system resilience to narrowband interference and channel distortion. Moreover, this results in a high level of system flexibility, since modulation parameters such as constellation size, coding rate, manipulation sequence class, encoding method, character interleaving type, etc. can be independently selected for each subchannel. Problematic issues that limit, in some cases, the use of OFDM, include the significant magnitude of the peak of the emitted OFDM signal. As is known, the peak factor (PF) is defined as the ratio of the maximum (peak) instantaneous power of a signal to its average power. Increasing this parameter adversely affects the complexity of the highfrequency path design from amplifiers to the antenna, leading to a decrease in the efficiency of high-frequency equipment, to an increase in non-linear distortions. Synchronization, channel estimation, radio resource management are just some of the problems associated with multi-carrier data technology. In spite of the fact that OFDM provides high efficiency of spectrum utilization due to orthogonal frequency multiplexing [2], its out-of-band radiation may be unacceptable if the use of back-band is not foreseen. In particular, in $4 \mathrm{G} \mathrm{LTE}$ about $10 \%$ of the dedicated bandwidth is reserved as a guard interval (also known as a cyclic prefix). This is quite a significant fee for a factor such as a spectrum resource. Frequency and time resources in OFDM are evenly divided into a number of elements of the same size for transmitting information [3]. In order to achieve orthogonality and to avoid inter-character or channel interference, it is necessary to ensure strict coordina- 
tion of the operation (in time and frequency) of the elements of the transmitting and receiving sides of the system. The synchronization procedure results in intensive signal transmission to achieve perfect synchronization, especially in the case of uplink transmission. Improper synchronization can lead to suboptimal system performance. Fifth-generation (5G) systems offer many advantages over earlier systems, such as: high data rates, ultra-reliable low latency, high spectral efficiency, high subscriber connectivity, and enhanced energy efficiency. To take advantage of 5G, experts in the field and academia have proposed new and effective technologies based on OFDM modulation [4]: window-OFDM, Multiple Frequency Filter Group (FBMC); orthogonal frequency division encoded channel (C-OFDM) technology; Universal Filtered Multi-Carrier (UFMC); filtered-OFDM, FC-FOFDM rapid-convolution system, etc. The filtration process is a proven and effective way of suppressing side lobes in OFDM. In the UFMC, filtering is applied to a block of sub-sequential subcarriers, which provides low out-of-band radiation. In multi-channel channels, UFMC is ineffective due to significant inter-character interference, which leads to suboptimal characteristics. In OFDM filtration systems, the available bandwidth is divided into many bands, enabling different sets of services to be implemented in different bands with signals filtered in the time domain accordingly. On the other hand, an approach based on filtering in the frequency domain was proposed, which has less computational complexity and increased flexibility compared to time domain filtering. The filter design is based on window optimization in the frequency domain, which balances the required minimum bandwidth attenuation, transition bandwidth, and error vector characteristics (EVM). OFDM is a type of frequency division multiplexing in which multiple subcarriers at adjacent frequencies are used in a single channel. The presence of multiple subcarriers in a single channel may create mutual interference, but due to the orthogonality of the subcarriers, this does not occur. For this reason, OFDM application maximizes the channel's spectral efficiency without interference. The spectrum of the OFDM system in the frequency domain is presented in Figure 1 [2].

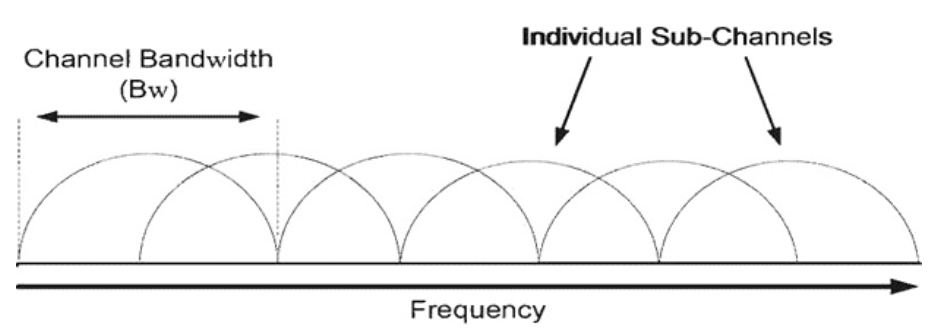

Fig. 1. OFDM frequency spectrum

\section{Receiver / transmitter architecture in OFDM system}

The classic OFDM transmitter and receiver model is shown in Fig. 2, $a$ - OFDM transmitter model, Fig. 2, $b$ - OFDM receiver. The transmitter converts the digital data that is to be transmitted to the corresponding amplitude and phase values, and then, using a backward Fourier transform (FFT), the digital data from the spectral representation is transformed into a time domain signal representation of the by adding a protective interval $(\mathrm{CI})$. Thus, the received signal data is subjected to frequency multiplexing. The reverse operation is performed on the receiver side, as shown in Fig. 2, $b$. When the modulated OFDM signal arrives at the receiver, the radio frequency signal is summed up with the main carrier and the $\mathrm{CO}$ is deleted. The signal spectrum is then converted to the frequency domain using a Fourier transform (FFT). Then the subcarrier phase and amplitude are extracted and demodulated back into the digital data. 


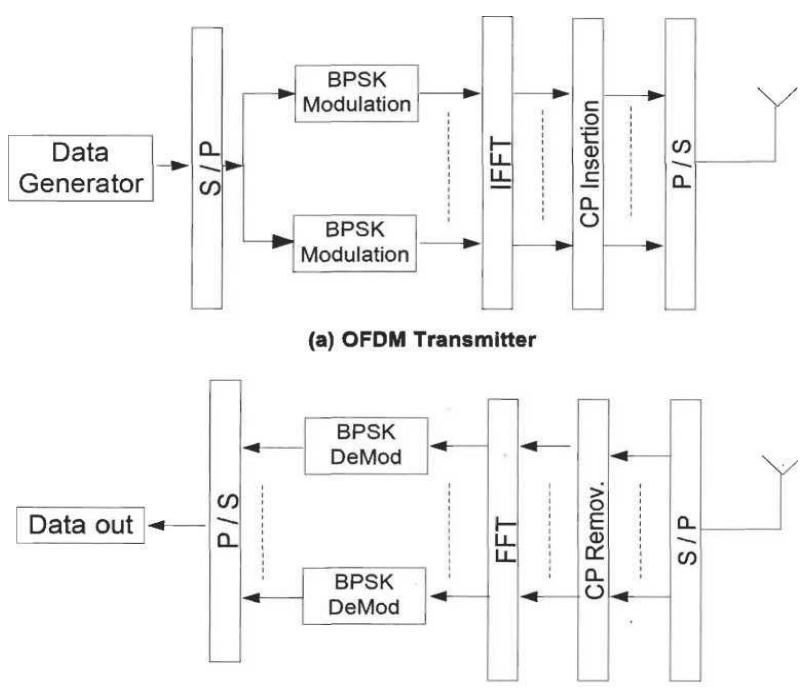

(b) OFDM Reciever

Fig. 2. Traditional OFDM system

In the transmitter, the output sequential stream of information bits is encoded by interferencetight code (according to LTE 3GPP TS 36.211 recommendation convolutional turbo codes are used with a base rate of 1/3), interspersed (P) and demultiplexed into $\mathrm{N}$ parallel sub-streams. Next, each of the streams is mapped to the symbol stream by phase pro-procedure (BPSK, QPSK, 8-PSK) or amplitude-phase quadrature modulation (QAM). When using BPSK modulation, a stream of binary numbers (1 and -1) is formed, with QPSK, 8-PSK, QAM a stream of complex numbers. In addition to the subcarriers on which the information is transmitted, service subcarriers are used. These include security intervals, pilots and additional service information to synchronize the receiver and transmitter and their operation modes. Pilots may have a fixed position on a subcarrier or a variable, with changes occurring from symbol to OFDM symbol in frames. Due to the insertion of interinterconnections under channels of sufficient length of the protective interval, the possibility of spectral overlap is eliminated. In this case, the inter-channel interference (between bit interference, ICI) decreases, the probability of a bit error decreases, and thus the bandwidth of the wireless access system increases. The multiplication operation on a set exponent with the corresponding subchannel frequency and then summing all the sub channels to generate an OFDM signal is very similar to the Fourier Inverse Transform operation. In this regard, to form the required OFDM-symbol is used the device SHZPF, which greatly simplifies the implementation of modulators. Maintaining orthogonality is necessary in order for the receiver to correctly recognize the information on the subcarriers. To do this, you must complete the following conditions: the receiver and the transmitter must be precisely synchronized; the analog components of the transmitter and receiver must be of very high quality; the channel should not be multipath. In this case, multipath radiation is almost inevitable in radio communication systems, which leads to distortion of the received signal. To eliminate this kind of obstruction, you must select a protection interval that should be longer than the maximum propagation delay in the channel. Thus, it is possible to eliminate more than six types of interference between channels (ie interference between subcarriers) and between adjacent transmission units (ie between symbolic interference). To reduce the out-of-band emission of signals, a window processing of a temporal signal with the use of a window of the type "raised cosine" is used. Further, digital-to-analog converters (DACs) convert into an analog view separately the true and imaginary components. After passing through the low pass filter, the signal is fed to a quadrature mixer, which transfers the useful spectrum of the OFDM signal to the carrier frequency. These signals are further summed up, amplified and the OFDM signal is generated. 


\section{The use of a cycle prefix}

In a wireless system, the radio signal in the transmission medium is reflected from different objects, causing multiple signals to be received at the receiver at different times. This phenomenon is known as multipath transmission. On the OFDM receiver side, the multipath propagation channel is represented as a distortion of the time at which the duration of each OFDM symbol increases [5]. As a result, the resulting symbols create obstacles to one another and form between symbolic obstacles [6]. Symbol rate for OFDM technology is much lower than for single carrier. For example, in a single-carrier system with BPSK modulation, the bit rate directly determines the transmission rate of sim-waves [7]. But in OFDM, the entire bandwidth is subdivided into $\mathrm{N}_{\mathrm{f}}$ subcarriers, which leads to $\mathrm{N}_{\mathrm{f}}$ - times a lower rate of symbol transmission than when transmitted from a single carrier. Thus, the effect of inter-character interference is reduced by multipath transmission from OFDM, which makes OFDM systems more resistant to ISI. The data transmission system can be improved by applying a buffer interval, which is a copy of a part of the transmitted signal of the OFDM symbol and this part is added to the beginning of the OFDM frame (Fig. 3). The use of a guard interval leads to an increase in the signal wavelength, but it significantly reduces the ISIs caused by multipath transmission [8-9].

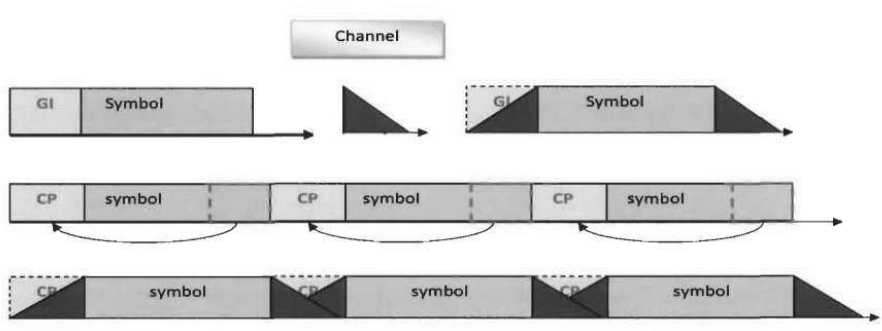

Fig. 3. Addition of CP to OFDM structure

With OFDM modulation, many subcarriers at adjacent frequencies are used in a single channel. This, in turn, can lead to mutual interference, but due to the orthogonality of the subcarriers, this does not happen [10]. Therefore, the application of OFDM to-maximize the spectral efficiency of the channel without interference. The spectrum of the OFDM system in the frequency domain is presented in Figure 3 [2].

\section{Channel subcarriers orthogonality}

As noted above, the subcarriers in OFDM systems are orthogonal. Thus, the sub-carriers are positioned as close as possible to one another, thereby increasing spectral efficiency. In other words, orthogonality provides simultaneous transmission to each subcarrier in the frequency space without interference [2] (Fig. 4). Thus, it is possible to detect signals on individual subcarriers in the receiving device. On the other hand, in a frequency-modulated conventional (FDM) system, such subcarrier overlap is impossible and, to avoid interference with the carriers, a protective band between the carriers is used. The OFDM multiuser version is OFDMA (Orthogonal Frequency Division Multiple Access) [5]. In this system, subsets of subcarriers are assigned to the individual user dynamically, using time or frequency division (Fig. 5), thus supporting simultaneous data transmission to multiple users. Using OFDMA, each user has his or her own unique set of subchannels (Fig. 5) and the base station can dynamically distribute subcarriers to users [4], for example, when a particular user has requested more resources. In essence, this means that this user may need higher radiation power, a large number of sub-channels, and appropriate modulation types. 


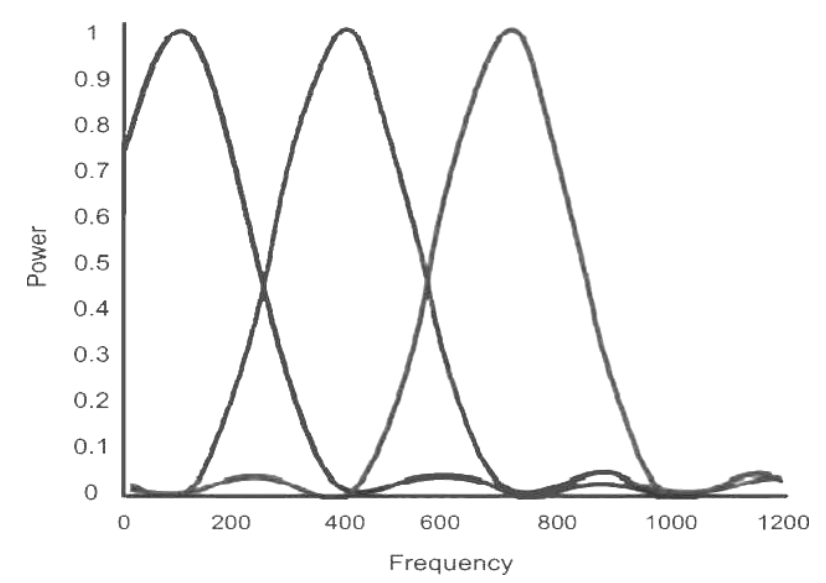

Fig. 4. Orthogonal intersection of the OFDM spectrum

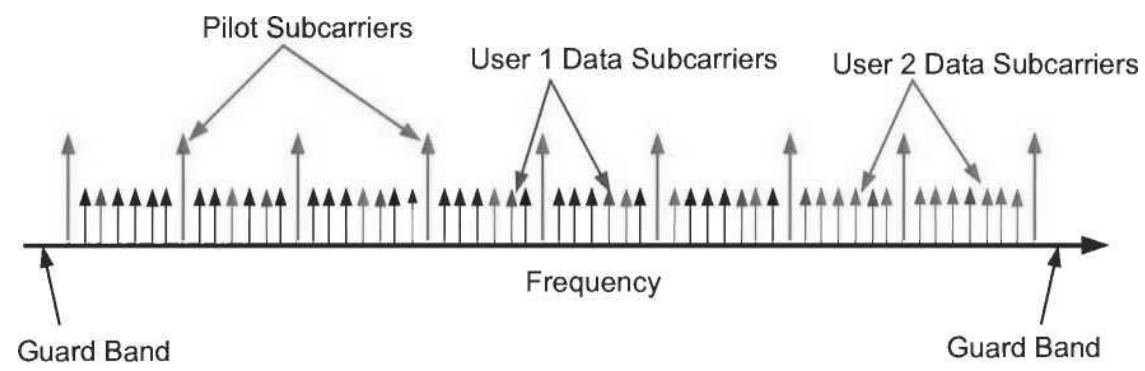

Fig. 5. Multiuser OFDM system

Thus, the main advantages of OFDMA include the following.

Resistance to fading.

Deployment flexibility in different frequency bands with small changes required for the radio interface.

Allows you to control channel or subchannel power.

By distributing carriers across the spectrum used, frequency diversity can be used.

Provides high signal quality when using a single carrier.

Mathematical models of OFDM and its versions

In the OFDM system in the converter (S/P) (Fig. 2) the serial information flow is transformed into $\mathrm{N}_{\mathrm{f}}$ parallel flows, where $\mathrm{N}_{\mathrm{f}}$ - the number of subcarriers before CP insertion. These parallel streams are then modulated using BPSK. With, every k symbol OFDM is given as [3]:

$$
X_{k}(t)=\sum_{n=0}^{N_{f-1}} S_{k, n} p(t-k T) e^{j 2 \pi \frac{n}{T} t},
$$

where T - duration of OFDM symbol, and

$$
S_{k}=\left[S_{k, 0}, S_{k, 1}, \ldots, S_{k, N_{f-1}}\right]^{T},
$$

$\mathrm{Nf}$ - parallel streams of data for one OFDM frame before inserting the CP,

$\mathrm{T}$ - the transposition operator, and $\mathrm{p}(\mathrm{t})$ is the impulse form used to form the symbols.

If we consider a rectangular pulse shape as a model:

$$
p(t)=\left\{\begin{array}{l}
1,0 \leq t \leq T \\
0, \text { otherwise }
\end{array}\right.
$$

and provided that each subcarrier and OFDM symbol is selected $\mathrm{N}_{\mathrm{f}}$ once per frame interval, the modulated signal (1) will look like: 


$$
X_{k}\left(\frac{m T}{N_{f}}\right)=\sum_{n=0}^{N_{f-1}} S_{k, n} e^{j 2 \pi n m / N_{f}}, m=0,1,2, \ldots, N_{f}-1
$$

Further, IFFT is used to obtain the modulated signal:

$$
X_{k}=N_{f} \operatorname{IFFT}\left(S_{k}\right)
$$

The thus obtained parallel stream of symbols after OFDM modulation is transformed into a sequential stream to which $\mathrm{CP}$ is added. The duration of IS to eliminate ISI should be greater than the channel delay. In the receiving device, the signal is converted from sequential to parallel, and the CP is removed, then FFT is used to demodulate and make decisions according to the type of modulation used.

\section{MIMO-OFDM systems}

In MIMO-OFDM systems, the wireless link is a transmitter and receiver system equipped with multiple antennas (Fig. 6). The main reason for the growing popularity of the MIMO-OFDM system is the ability to provide high quality signal and a high data rate. This is ensured by combining the signals on the transmit $\left(\mathrm{T}_{\mathrm{X}}\right)$ antennas at one end and the receive $\left(\mathrm{R}_{\mathrm{X}}\right)$ antennas at the other end. The resulting quality and performance improvements result in the communication lines being able to be used to significantly improve the quality of wireless network service.

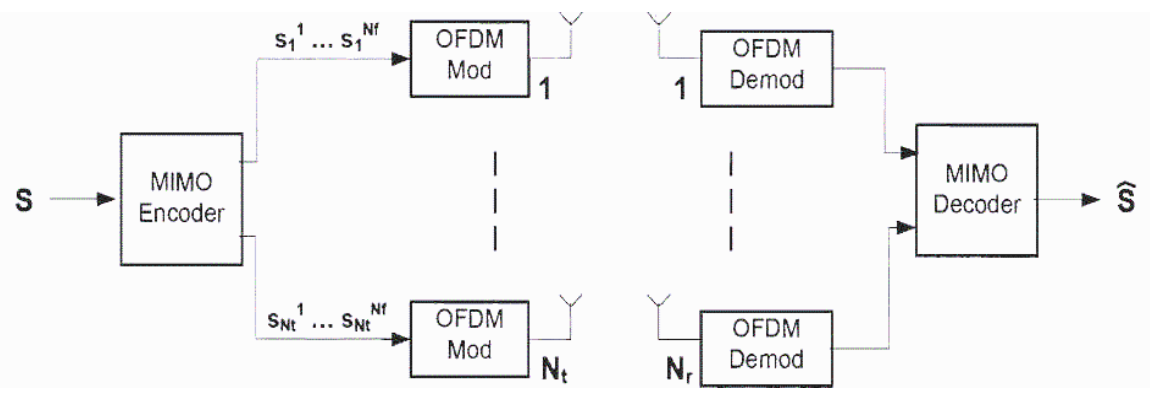

Fig. 6 A simplified block diagram of the MIMO-OFDM system

The MIMO-OFDM system involves the use of $\mathrm{N}_{\mathrm{t}}$ transmitting antennas and $\mathrm{N}_{\mathrm{r}}$ receiving antennas (Fig. 6). Initially, the input bitstream is displayed in several data characters using certain types of modulation, such as BPSK. Then block of $\mathrm{N}_{\mathrm{s}}$ character data $\left[\mathrm{s}_{1}, \mathrm{~s} 2, ., ., \mathrm{s}_{\mathrm{Ns}}\right]$ transforms into a codeword matrix $\mathrm{S}$ of size $\mathrm{TXN}_{\mathrm{t}}$, which then in $\mathrm{T}$ frames after implementation of OFDM modulation using $\mathrm{N}$ antennas will be transmitted to the communication channel. In this case, each frame consists of $\mathrm{N}_{\mathrm{f}}$ subcarriers. Exactly, $\mathrm{S}_{1 \mathrm{j}}, \mathrm{S}_{2 \mathrm{j}}, \ldots, \mathrm{S}_{\mathrm{Tj}}$ code words will be transmitted with each $\mathrm{j}_{\mathrm{th}}$ transfer antennas in the form of $1,2, \ldots$ T OFDM frames respectively. Code word $S_{n j}$ means the vector of length $\mathrm{N}_{\mathrm{f}}$, for all $\mathrm{j}=1,2, \ldots, \mathrm{N}_{\mathrm{t}}$ and $\mathrm{n}=1,2, \ldots, \mathrm{T}$. The codeword matrix $\mathrm{S}$ can be expressed as:

$$
S=\left(\begin{array}{ccc}
S_{1}^{1} & \cdots & S_{1}^{T} \\
\vdots & \ddots & \vdots \\
S_{N_{t}}^{1} & \cdots & S_{N_{t}}^{T}
\end{array}\right)
$$

After adding a cyclic prefix in all OFDM frames, $S_{\mathrm{nj}}$ will be transmitted from the transmitting antenna at $n_{\text {th }}$ OFDM frame. We define $X$ as a subset of $S$, which represents an array of characters transmitted from all transmit antennas within a single OFDM frame, of dimension $\mathrm{N}_{\mathrm{f}} \mathrm{X} \mathrm{N}_{\mathrm{t}}$ : 


$$
X=\left(\begin{array}{ccc}
S_{1}^{1} & \cdots & S_{1}^{N_{f}} \\
\vdots & \ddots & \vdots \\
S_{N_{t}}^{1} & \cdots & S_{N_{t}}^{N_{f}}
\end{array}\right)
$$

Expression (7) can be written as follows:

$$
X^{\left(k=1: N_{f}\right)}=\left[\begin{array}{c}
X_{1}{ }^{\left(k=1: N_{f}\right)} \\
X_{2}{ }^{\left(k=1: N_{f}\right)} \\
\vdots \\
X_{N_{t}}{ }^{\left(k=1: N_{f}\right)}
\end{array}\right] .
$$

Here vector $X_{j}{ }^{\left(k=1: N_{f}\right)}$ represents all characters transmitted from antenna $\mathrm{j}$ using $\mathrm{N}_{\mathrm{f}}$ subcarriers. At the receiving station in the MIMO system, the received signals perform the procedures opposite to those implemented in the transmitting device. The protective terminal is removed and the FFT is applied and the data is then fed to the decoder. The resulting vector can be represented as:

$$
Y^{\left(k=1: N_{f}\right)}=\left[\begin{array}{c}
Y_{1}^{\left(k=1: N_{f}\right)} \\
Y_{2}^{\left(k=1: N_{f}\right)} \\
\vdots \\
Y_{N_{r}}{ }^{\left(k=1: N_{f}\right)}
\end{array}\right]
$$

Consider only the subcarrier $(\mathrm{k}=1)$. Then equation (9) can be represented as:

$$
\mathrm{Y}=\mathrm{HX}+\mathrm{v}
$$

where: Y - obtained vector, with dimension $\mathrm{Nr}$;

$\mathrm{H}-\mathrm{Nr} \times \mathrm{Nt}$ - complex signal propagation matrix, whose value is constant for the length of the transmitted frame (ie quasi-static channel) and is known in the receiver (for example, by transmitting test sequences). It is assumed that the statistics of the channel transmitter of the matrix $\mathrm{H}$ can be described by the attenuation statistics, namely: Rayleigh attenuation, Rice attenuation or AWGN. In addition, it is assumed that the elements $\mathrm{H}$ have a variance equal to one, or, in other words, the average gain of the channel $\mathrm{P}_{c}$ is normalized to one. Exposure to radio frequencies emitted by a transmitter by a counter-station may impair the characteristics of the receiving station's (AU) receiver. The level of interference power at the input of the AU receiver is usually set as [8] - [11]:

$$
I=P_{T}-L_{F L . T}+G_{T}+G_{R}-L_{F L . R}-L_{P O L}-L_{P}-L_{F D R},
$$

where $\mathrm{P}_{\mathrm{T}}$ - transmitter output power in $\mathrm{dB} \cdot \mathrm{W}$;

$\mathrm{L}_{\mathrm{FL}, \mathrm{T}}$ - losses in the feeder line between the output of the transmitter and the input of the transmitting antenna;

$\mathrm{G}_{\mathrm{T}}$ and $\mathrm{G}_{\mathrm{R}}$ - gain of transmitting and receiving antennas;

$\mathrm{L}_{\mathrm{FL}, \mathrm{R}}$ - losses in the feeder line between the output of the receiving antenna and the input of the receiver;

$\mathrm{L}_{\mathrm{POL}}$ - loss due to mismatch of polarization of the receiving antenna;

$\mathrm{L}_{\mathrm{P}}$ - propagation loss (including interference loss) between the transmitting and receiving antennas;

$\mathrm{L}_{\mathrm{FDR}}$ - frequency-dependent deviation (FDR) losses.

FDR is a measure of the deviation caused by the selectivity curve of the receiver in the spectra of unwanted transmitter radiation, and can be represented as: 


$$
L_{F D R}=10 \log _{10}\left(\frac{\int_{-\infty}^{\infty} \Phi(f) d f}{\int_{-\infty}^{\infty} \Phi(f) \Psi(f-\Delta f) d f}\right),
$$

where $\Phi(f)$ - power spectral density (PSD) of a complex equivalent representation of a fundamental frequency band (or complex envelope) of a true interference signal;

$\Psi(f)$ - normalized frequency response of the receiver;

$\Delta f$ - frequency shift between the counter station transmitter and the AC receiver [11]. For signal signature $\Psi(f-\Delta f)$, if $\Delta f-\frac{W_{v}}{2} \leq f \leq \Delta f+\frac{W_{v}}{2}$, the expression for FDR is simplified to the form:

$$
L_{F D R}=10 \log _{10}\left(\frac{\int_{-\infty}^{\infty} \Phi(f) d f}{\Delta f+\frac{W_{v}}{2}} \int_{\Delta f-\frac{W_{v}}{2}}^{\int^{2}} \Phi(f) d f\right),
$$

where $\mathrm{W}_{\mathrm{v}}$ - the bandwidth of the channel of the receiver AC.

The PSD signal area is equal to the signal power, and thus we obtain an expression to calculate the transmitter output power: $P_{T=10 \log _{10}}\left(\int_{-\infty}^{\infty} \Phi(f) d f\right)$.

As shown in [13], FDR mainly depends on the interference power spectral density (PSD).

\section{CP-OFDM systems}

The complex envelope signal transmitted in CP-OFDM technology [11] can be expressed as:

$$
s(t)=\sum_{n=-\infty}^{\infty} \sum_{k=0}^{N-1} c_{n, k} p\left(t-n\left(T_{s}+T_{g}\right)\right) e^{-j 2 \pi k \frac{1}{n}\left(T_{s}+T_{g}\right)},
$$

where $c_{n, k}$ - complex data symbol modulated on the k-th subcarrier of the $n$-th OFDM symbol,

$\mathrm{p}(\mathrm{t})$ - window of impulse formation,

$\mathrm{T}_{\text {tot }}=\mathrm{T}_{\mathrm{s}}+\mathrm{T}_{\mathrm{g}}$ - total character length,

$\mathrm{T}_{\mathrm{s}}$ and $\mathrm{T}_{\mathrm{g}}$ - the length of the data symbol and the security interval, respectively.

Assuming that the complex signals on each subcarrier are statistically independent and mutually orthogonal, the expression for the power spectral density of the OFDM signal with arbitrary pulse formation is given as [11] - [13]:

$$
\Phi_{s}(f)=\frac{P_{s}}{T_{\text {tot }}} \sum_{k=0}^{N-1}\left|P\left(f-\frac{k}{T_{s}}\right)\right|^{2},
$$

where $\mathrm{P}_{\mathrm{S}}$ - data symbols dispersion $\mathrm{c}_{\mathrm{n}, \mathrm{k}}$, as well as the power of one of the OFDM subcarriers;

$1 / \mathrm{T}_{\mathrm{S}}$ distance between subcarriers;

$\mathrm{P}(\mathrm{f})$ - Fourier transform impulse forming window. 
Adding the representation of the rectangular momentum form to (14), we obtain:

$$
\begin{array}{r}
p(t)=\Pi\left(\frac{t-\frac{T_{t o t}}{2}}{T_{t o t}}\right), \\
\partial e \Pi\left(\frac{t}{T_{t o t}}\right)=\left\{\begin{array}{l}
0, \text { if }|t|>\frac{T_{t o t}}{2} \\
\frac{1}{2}, \text { if }|t|=\frac{T_{t o t}}{2} \\
1, \text { if }|t|<\frac{T_{t o t}}{2}
\end{array}\right.
\end{array}
$$

Using the shift time property of the Fourier transform, $|\mathrm{P}(\mathrm{f})|^{2}$ is expressed as

$$
|P(f)|^{2}=|\mathfrak{I}\{p(t)\}|^{2}=\left|\mathfrak{I}\left\{\Pi\left(\frac{t}{T_{t o t}}\right)\right\} e^{-j \pi_{\text {tot }}}\right|^{2}=\left|\mathfrak{I}\left\{\Pi\left(\frac{t}{T_{t o t}}\right)\right\}\right|^{2}=T_{t o t}^{2} \sin c^{2}\left(T_{t o t} f\right)
$$

where $\sin c-\mathrm{a}$ function that is defined as $\sin \mathrm{c}(\mathrm{x})=\sin (\pi \mathrm{x}) / \pi \mathrm{x}$, if $\mathrm{x} \neq 0$, otherwise - equals one.

Using (15) and (17), the power spectral density (PSD) of CP-OFDM with a rectangular pulse shape is defined as:

$$
\Phi_{s}^{(C P)}(f)=P_{s} T_{t o t} \sum_{k=0}^{N-1}\left\{\sin c\left[\left(f-\frac{k}{T_{s}}\right) T_{t o t}\right]\right\}^{2} .
$$

Let FDR be the measure of the deviation determined by the selectivity curve of the receiver on the spectrum of unwanted transmitter radiation. Applying (13) and (18), we obtain an expression to calculate the LFDR of CP-OFDM technology:

$$
L_{F D R}^{(C P)}=P_{T}-10 \log _{10}\left(\frac{P_{s}}{\pi} \sum_{k=0}^{N-1}\left[\frac{\sin ^{2}\left(f_{k}^{-}\right)}{f_{k}^{-}}-\frac{\sin ^{2}\left(f_{k}^{+}\right)}{f_{k}^{+}}-\operatorname{Si}\left(2 f_{k}^{-}\right)+\operatorname{Si}\left(2 f_{k}^{+}\right)\right]\right),
$$

where

$$
\begin{aligned}
& S i(x)=\int_{0}^{x} \frac{\sin t}{t} d t \\
& f_{k}^{+}=\pi T_{t o t}\left(\Delta f+\frac{W_{v}}{2}-\frac{k}{T_{s}}\right) \\
& f_{k}^{-}=\pi T_{t o t}\left(\Delta f-\frac{W_{v}}{2}-\frac{k}{T_{s}}\right) .
\end{aligned}
$$

\section{Windowed-OFDM systems}

For the suppression of out-of-band radiation in the OFDM window system, window-time functions, for example, the function of the increased co-sine, are used to generate momentum. $\mathrm{w}_{\mathrm{rc}}(\mathrm{t}), \mathrm{du}-$ ration $\mathrm{T}_{\mathrm{w}}=\mathrm{T}_{\text {tot }}+\mathrm{T}_{\text {tr }}$ kind:

$$
p(t)=w_{r c}\left(t-\frac{T_{t o t}}{2}\right)
$$




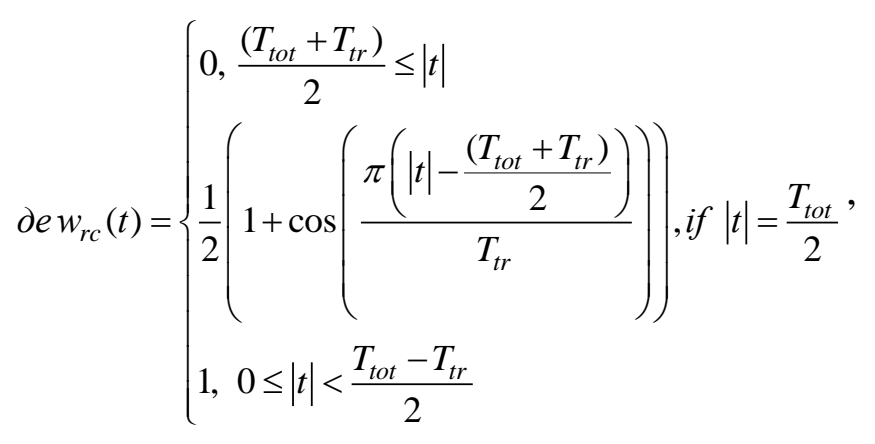

$\mathrm{T}_{\text {tr }}$ - signal transmission time.

From (15) and the Fourier transform (20) the expression for the power spectral density of the OFDM window can be represented as:

$$
\Phi_{s}^{(W)}(f)=P_{s} T_{t o t} \sum_{k=0}^{N-1}\left\{\sin c\left[\left(f \times \frac{k}{T_{s}}\right) T_{t o t}\right] \times \frac{\cos \left(\pi T_{t r}\left(f-\frac{k}{T_{s}}\right)\right)}{1-4 T_{t r}^{2}\left(f-\frac{k}{T_{s}}\right)^{2}}\right\}^{2} .
$$

It follows from (21) that the expression for the FDR of the OFDM window is defined as:

$$
L_{F D R}^{(C P)}=P_{T}-10 \log _{10}\left[P_{s} T_{t o t} \sum_{k=0}^{N-1} \int_{\Delta f-\frac{W_{v}}{2}}^{\Delta f+\frac{W_{v}}{2}}\left\{\sin c\left(\left(f-\frac{k}{T_{s}}\right) T_{t o t}\right)^{2} \times \frac{\cos ^{2}\left(\pi T_{t r}\left(f-\frac{k}{T_{s}}\right)\right)}{\left(1-4 T_{t r}{ }^{2}\left(f-\frac{k}{T_{s}}\right)^{2}\right)^{2}}\right\} d f\right] .
$$

The latter expression can be implemented using software for settlement calculations. As follows from expression (22), the FDR of the OFDM window is affected only by the parameters: $\mathrm{T}_{\mathrm{tr}}$, $\mathrm{T}_{\mathrm{s}}$, and $\mathrm{T}_{\text {tot. }}$.

\section{Filtered OFDM systems}

The filtered $x(t)$ OFDM signal is formed by passing the $s(t)$ signal CP-OFDM (6) through the spectrum forming filter. Thus, $x(t)$ is given by the convolution $s(t)$ and the impulse response of the filter $\mathrm{h}(\mathrm{t})$ in this way:

$$
x(t)=s(t) \cdot h(t)
$$

We apply a spectrum forming filter [9], which is based on the truncation of the basic filter. The truncation is performed by applying the window-time function $w(t)$ to the impulse response of the main filter $g(t)$. The impulse response of a truncated filter is defined as

$$
h(t)=g(t) \cdot w(t) .
$$

Suppose a function $\sin \mathrm{c}$ for $g(t)=W_{g} \sin c\left(W_{g} t\right)$, has a frequency response $W_{g}=\Pi\left(\frac{f}{W_{g}}\right)$. To suppress out-of-band radiation, the base filter truncates by using window-time functions such as the Henning, Hamming, and Blackman windows [11]. For example, a Henning window of $\mathrm{T}_{\mathrm{w}}$ duration is defined as: 


$$
w(t)=\left\{\begin{array}{ll}
\frac{1}{2}+\frac{1}{2} \cos \left(\frac{2 \pi|t|}{T_{w}}\right),|t| \leq \frac{T_{w}}{2} \\
0,|t|>\frac{T_{w}}{2}
\end{array} .\right.
$$

Filtered-OFDM PSD signal is given as

$$
\begin{aligned}
& \Phi_{x}(f)=\frac{P_{s} T_{\text {tot }}}{16 \pi^{2}}\left[2 \operatorname{Si}\left(\pi f_{u}\right)-2 \operatorname{Si}\left(\pi f_{l}\right)+\operatorname{Si}\left(\pi-\pi f_{l}\right)-\operatorname{Si}\left(\pi-\pi f_{u}\right)+\operatorname{Si}\left(\pi+\pi f_{u}\right)-\operatorname{Si}\left(\pi+\pi f_{l}\right)\right]^{2} \\
& \times \sum_{k=0}^{N-1}\left\{\operatorname{Sin} c\left[\left(f-\frac{k}{T_{s}}\right) T_{t o t}\right]\right\}^{2}
\end{aligned}
$$

where $\mathrm{f}_{\mathrm{u}}=\mathrm{T}_{\mathrm{w}}\left(\mathrm{f}+\mathrm{W}_{\mathrm{g}} / 2\right)$, and $\mathrm{f}_{\mathrm{l}}=\mathrm{T}_{\mathrm{w}}\left(\mathrm{f}-\mathrm{W}_{\mathrm{g}} / 2\right)$.

It should be noted that the PSD of the filtered OFDM signal can be found in quasi-closed form, since $S_{i}(x)$ can be estimated as easily as the basic trigonometric function using numerical calculation software. Using (26), the FDR of filtered-OFDM is defined as:

$$
L_{F D R}^{(F)}=P_{T}-10 \log _{10}\left[\frac{P_{S} T_{\text {tot }}}{16 \pi^{2}} \sum_{k=0}^{N-1} \int_{\Delta f-W_{v}}^{\Delta f+W_{v}} \sin c\left(\left(f-\frac{k}{T_{s}}\right) T_{t o t}\right)^{2} \times\left\{\begin{array}{l}
2 S i\left(\pi f_{u}\right)-2 S i\left(\pi f_{l}\right)+S i\left(\pi-\pi f_{l}\right) \\
-S i\left(\pi-\pi f_{l}\right)+S i\left(\pi+\pi f_{u}\right)-S i\left(\pi+\pi f_{u}\right)
\end{array}\right\}^{2} d f\right] .
$$

This expression can be implemented programmatically for numerical calculations [11]. Analysis of the latter expression shows that the FDR of the filtered OFDM is affected by the parameters: $\mathrm{T}_{\mathrm{w}}, \mathrm{Ts}, \mathrm{T}_{\mathrm{tot}}$, and $\mathrm{W}_{\mathrm{g}}$. This is important in terms of controlling inter-character and inter-channel interference in the development of OFDM system architecture.

\section{Conclusions}

The use of signals with orthogonal frequency division channels, allows to sub-increase not only the information capacity of the system in conditions of multipath propagation with a limited bandwidth, but also the speed of data transmission, bringing it closer to the bandwidth of the channel, increase the transmission secrecy and noise immunity, the effects of multipath propagation (provided the appropriate shielding interval is used), increase the spectral efficiency of the system. The article analyzes the systems of OFDM and a number of systems, which basically contain procedures of orthogonal time-division of channels and multiplexing. Mathematical models of OFDM signals are obtained, describing the basic stages of transformations that are performed to obtain such signals and estimating the properties of the signals. The obtained results can be used in the construction of secure information and communication systems, for which the primary tasks are to provide the necessary indicators of noise immunity (noise immunity of receiving signals, system operation), information security, resistance to interference, spectral and energy efficiency.

\section{References:}

1. Замула А.А., Морозов В.Л. Принципы применения технологии ортогонального частотного разделения каналов и мультиплексирования (OFDM) для построения современных беспроводных коммуникационных систем. Проблеми інформатизації // Матеріали шостої міжнародної науково-технічної конференції, 14 - 16 листопада 2018. - С. 23-24.

2. OFDM and Multi-Channel Communication Systems, National Instruments Measurement Fundamentals series, Publish Date: Feb 02, 2012.

3. Rodger Ziemer and William Tranter, Principles of Communications - Systems Modulation and Noise, fifth edition, John Wiley and Sons Ltd, NJ, 2002.

4. K. B. Letaief and Y. Zhang. Dynamic Multiuser Resource Allocation and Adaptation for Wireless Systems // IEEE Wireless Commun., vol. 13, no. 4, Aug. 2006, pp. 38-47.

5. S. Srikanth, V. Kumaran, C. Manikandan et al. Orthogonal Frequency Division Multiple Access: is it the multiple access system of the future. AU-KBC Research Center, Anna University, India. 
6. A. D. S. Jayalath and C. Tellambura, "Reducing the out-of-band radiation of OFDM using an extended guard interval // IEEE 54th Veh. Technol. Conf. (VTC Fall), Atlantic City, NJ, USA, vol. 2, Oct. 2001, pp. 829-833.

7. C. Liu and F. Li. Spectrum modelling of OFDM signals for WLAN // Electron. Lett., vol. 40, no. 22, pp. 14311432, Oct. 2004.

8. Procedures for Determining the Potential for Interference Between Radars Operating in the Radiodetermination Service and Systems in Other Services, document ITU-R Rec. M.1461-2, Jan. 2018.

9. Frequency and Distance Separations, document ITU-R Rec. SM.337-6, Oct. 2008.

10. S. H. Raghavan and H. Chew. Frequency-dependent rejection, spectral separation coefficient, and interference analysis // IEEE Aerosp. Conf., Big Sky, MT, USA, Mar. 2018, pp. 1-10.

11. Park, Jaedon \& Lee, Eunhyoung \& Park, Sung-Ho \& Sabogu-Sumah, Raymond \& Pyo, Seongmin \& Jo, HanShin. Modeling and Analysis on Radio Interference of OFDM Waveforms for Coexistence Study // IEEE Access. PP. 11. 10.1109/ACCESS.2019.2896280.

12. T. Levanen, J. Pirskanen, K. Pajukoski, M. Renfors, and M. Valkama. Transparent Tx and Rx waveform processing for $5 \mathrm{G}$ new radio mobile communications // IEEE Wireless Commun., vol. 26, no. 1, pp. 128-136, Feb. 2019. doi: 10.1109/MWC.2018.1800015.

13. Qualcomm. (2017). Spectrum for $4 \mathrm{G}$ and 5G. [Online]. Available: https://www.qualcomm.com/media/documents/files/spectrum-for-4ga.

Kharkiv National V.N. Karazin University;

JSC "Institute of Information Technologies"

Received 07.08.2019 